\title{
Dimension changes in a solid containing anisotropic defects
}

\author{
A M Stoneham \\ Theoretical Physics Division, AERE, Harwell, Didcot, Berks.
}

MS received 18 October 1972

\begin{abstract}
Aligned anisotropic defects in a solid cause different changes in macroscopic dimensions and in lattice parameters along the different crystal axes. A simple general method is given for relating these differences to the microscopic forces associated with the individual defects. Explicit results are given for isotropic, cubic and hexagonal host lattices.
\end{abstract}

\section{Introduction}

When an asymmetric defect is placed in a solid, the mean lattice parameters, external dimensions, and shape of the solid are changed. Experimentally, one can measure the change in total volume and the changes in mean length along three orthogonal directions. In principle more anisotropic effects can be detected, but they are largely cancelled out by the combined effects of the many defects actually present. Here we concentrate on the differences among the fractional changes in dimension along the different crystal axes from the presence of the anisotropic defect. Since we are concerned solely with differences, there is no distinction between predictions for lattice parameter measurements and for macroscopic dimension measurements. The usual terms arising from the creation of new lattice sites (eg Simmons and Balluffi 1960) cancel out exactly. The method we use is similar to Temkin's (1970) earlier treatment of the volume change due to a defect.

\section{General method}

Consider a block of material with flat orthogonal faces. When an anisotropic defect is placed in the crystal, the effective forces $F_{i}$ exerted on the host atoms $i$ lead to lattice distortion. The position of the defect in the host is not important provided the $\boldsymbol{F}_{i}$ have a finite range which does not reach the surface. It will appear that the only position dependence occurs because the defect forces should be calculated at the relaxed atomic configuration, and the degree of relaxation is modified when the defect is near the surface. This dependence is usually negligible. The axes of the defect need not coincide with those of the (macroscopic) specimen, nor with those of the (microscopic) host lattice structure. The components of the $\boldsymbol{F}_{i}$ will always be referred to the (microscopic) lattice axes.

We now calculate $\left(\delta_{\| !}-\delta_{\perp}\right)$, where $\delta_{\mid 1}$ is the increase in mean length of the specimen along one axis, and $\delta_{\perp}$ is the average of the increases along the two perpendicular axes. In general the displacements $\delta_{I}$ normal to the $I$ faces vary over the face; $\delta_{! !}$and $\delta_{\perp}$ are 
surface averages of the $\delta_{I}$. For convenience we may relate $\delta_{\eta}$ and $\delta_{\perp}$ to the fractional changes in length, $x_{\| !}$and $x_{\perp}$. If $A_{I}$ is the area of face $I$ and $V$ is the crystal volume, then for the three orthogonal directions:

$$
\begin{aligned}
& x_{\| !}=\frac{A_{\|}}{V} \delta_{\|} \\
& x_{\perp 1}=\frac{A_{\perp 1}}{V} \delta_{\perp 1} \\
& x_{\perp 2}=\frac{A_{\perp 2}}{V} \delta_{\perp 2} .
\end{aligned}
$$

We shall also use $x_{\perp} \equiv\left(x_{\perp 1}+x_{\perp 2}\right) / 2$.

\section{I. The reciprocity theorem}

External forces $\boldsymbol{P}$ due to applied uniaxial stresses cause displacements $\boldsymbol{U}$ in the perfect solid. In the absence of applied stresses, the defect forces $\boldsymbol{F}$ give surface displacements $\boldsymbol{\delta}_{I}$. The Betti reciprocity theorem (Betti 1872, Love 1944), valid for any harmonic system, allows us to relate these two sets of forces and displacements. The theorem states that:

$$
\sum_{I} P_{I} \cdot \delta_{I}=\sum_{i} F_{i} \cdot U_{i}
$$

Since the stresses $\boldsymbol{P}$ are uniform over the surfaces, only the mean displacements $\delta_{i}$ or $\delta_{\perp}$ contribute to the left hand side.

Suppose a pressure $-2 P$ is applied to the faces normal to a chosen axis, and $+P$ to the other four faces. Positive values indicate compression. Then the left hand side of (2) is:

$$
\begin{aligned}
\sum_{I} P_{I} \cdot \delta_{I} & =2 P A_{\|} \delta_{\|}-P A_{\perp 1}-P A_{\perp 2} \delta_{\perp 2} \\
& =2 \operatorname{VP}\left(x_{\mid '}-x_{\perp}\right) .
\end{aligned}
$$

The displacements $U_{i}$ associated with the uniaxial stress can be related to e the uniform strain throughout the specimen, by

$$
U_{\alpha}\left(R_{i}\right)=\sum_{\beta} e_{\alpha \beta}\left(R_{i \beta}+\gamma_{i \beta}\right)
$$

where $\boldsymbol{R}_{i}$ gives the position relative to the defect, $\gamma_{i}$ gives the internal strain, and $e_{\alpha \beta} \equiv\left(\partial U_{\alpha} / \partial R_{\beta}+\partial U_{\beta} / \hat{c} \boldsymbol{R}_{\alpha}\right) / 2$. Thus the right-hand side of (2) takes the form:

$$
\begin{aligned}
\sum_{i} F_{i} \cdot \boldsymbol{u}_{i} & =\sum_{i} \sum_{\alpha, \beta} F_{i \alpha} e_{\alpha \beta}\left(R_{i \beta}+\gamma_{i \beta}\right) \\
& =P \sum_{i} \sum_{\alpha, \beta} F_{i \alpha}\left(e_{\alpha \beta} / P\right)\left(R_{i \beta}+\gamma_{i \beta}\right) .
\end{aligned}
$$

Here $e_{\alpha \beta} / P$ is, of course, independent of $P$ and merely a combination of the host elastic constants. Combining (2), (3) and (5) we obtain:

$$
\left(x_{\|}-x_{\perp}\right)=\frac{1}{2 V} \sum_{\alpha} \sum_{\beta} \sum_{i} F_{i \alpha}\left(e_{\alpha \beta} / P\right)\left(R_{i \beta}+\gamma_{i \beta}\right) .
$$

So far we have considered a single defect only. If there is a density of $\rho \equiv N / V$ defects per unit volume, all with parallel orientations, then their contributions to (6) simply add. The only change is that the $(1 / 2 V)$ factor becomes $(\rho / 2)$. If there are several different 
orientations, then (6) may be summed for each orientation separately; these contributions are then added.

\section{Examples of dimension changes}

It is completely straightforward to use equation (6). One axis is singled out as the axis by the experiment under consideration. The uniform strain induced by pressures $-2 P$ on the faces corresponding to this axis and by $P$ on the others is then calculated by ordinary elasticity theory. Equation (6) can then be used directly. We shall assume zero internal strain in these examples, although it is trivial to extend the theory to this case.

\subsection{Force dipole in an isotropic medium}

Choose the $\|$ axis to have direction cosines $(L, M, N)$, and let the two $\perp$ axes be $(l, m, n)$ and $(\lambda, \mu, \nu)$. The strain components are:

$$
\begin{aligned}
& e_{x x}=\frac{P}{2 \mu}\left(2 L^{2}-l^{2}-\lambda^{2}\right) \\
& \left.e_{x z}=\frac{P}{\mu} 2 L N-\ln -\lambda \mu\right)
\end{aligned}
$$

with other components related by symmetry. $\mu$ is the rigidity modulus.

If the force dipole is parallel to the chosen axis, it may be represented by forces $\pm F(L, M, N)$ at sites $\pm(d / 2)(L, M, N)$. When the density of aligned dipoles is $\rho$, the fractional dimension change is

$$
x_{\|}-x_{\perp}=\frac{\rho}{2} \frac{F d}{\mu} .
$$

As expected, there is no dependence on $(L, M, N)$.

\subsection{Defects in a cubic host}

We consider three choices of axis; (100), (111) and (110). The results given are valid for any defect symmetry, although it is natural to adopt a sample axis $(L, M, N)$ for defects with symmetry axes $(L, M, N)$. The reason is most easily seen for force dipoles. If one chooses (111) dipoles, then from reasons of symmetry alone they give no contribution to the relative changes in length the $(100),(010)$ and $(001)$ directions, irrespective of the degree of alignment.

In all cases, the correct isotropic limit is achieved by putting $c_{44}=\left(c_{11}-c_{12}\right) / 2=\mu$.

3.2.1 Tetragonal case: (001) axis. The faces are (001), (010) and (100). The nonzero strain components are:

$$
e_{z z}=-2 e_{x x}=-2 e_{y y}=\frac{2 P}{\left(c_{11}-c_{12}\right)}
$$


and the fractional displacements from aligned defects satisfy

$$
\left(x_{\|}-x_{\perp}\right)=\frac{\rho}{2\left(c_{11}-c_{12}\right)} \sum_{i}\left\{-R_{x i} F_{x i}-R_{y i} F_{y i}+2 R_{z i} F_{z i}\right\} .
$$

3.2.2. Trigonal case: (111) axis. The faces are (111), (110) and (112). The nonzero strain components are:

$$
e_{x y}=e_{y z}=e_{z x}=\frac{P}{c_{44}}
$$

The fractional displacements from aligned defects satisfy:

$$
\left(x_{\| !}-x_{\perp}\right)=\frac{\rho}{4 c_{44}} \sum_{i}\left\{F_{x i}\left(R_{y i}+R_{z i}\right)+F_{y i}\left(R_{x i}+R_{z i}\right)+F_{z i}\left(R_{x i}+R_{y i}\right)\right\}
$$

3.2.3. (110) axis. The faces are (110)(110) and (001). In this case $x_{\perp 1}$ and $x_{\perp 2}$ are not equal. The nonzero strains are

$$
\begin{aligned}
& 2 e_{x x}=2 e_{y y}=-e_{z z}=\frac{\frac{1}{2} P}{\left(c_{11}-c_{12}\right)} \\
& e_{x y}=\frac{3 P}{4 c_{44}} .
\end{aligned}
$$

The difference in fractional mean parallel and perpendicular displacements due to aligned defects is:

$$
\left(x_{1 !}-x_{\perp}\right)=\frac{\rho}{4\left(c_{11}-c_{12}\right)} \sum_{i}\left\{F_{x i} R_{x i}+F_{y i} R_{y i}-2 F_{z i} R_{z i}\right\}+\frac{3 \rho}{8 c_{44}} \sum_{i}\left\{F_{x i} R_{y i}+F_{y i} R_{x i}\right\}
$$

\subsection{Defects in a hexagonal host lattice}

The chosen axis here is the hexagonal axis. The results obtained are relevant for defects in graphite, for example, and, since they concentrate on $x_{\perp}$ rather than $x_{\perp 1}$ and $x_{\perp 2}$, may be used for pyrolytic graphite as well as for single crystals. The hexagonal lattice is isotropic in the basal plane.

The nonzero strains are

$$
\begin{aligned}
& e_{x x}=e_{y y}=\gamma_{\perp} P \\
& e_{z z}=2 \gamma_{\|} P
\end{aligned}
$$

where

$$
\begin{aligned}
& \gamma_{\perp}=\frac{c_{33}+2 c_{13}}{c_{33}\left(c_{11}+c_{12}\right)-2 c_{13}^{2}} \equiv s_{11}+s_{12}-2 s_{13} \\
& \gamma_{\|}=\frac{c_{11}+c_{12}+c_{13}}{c_{33}\left(c_{11}+c_{12}\right)-2 c_{13}{ }^{2}} \equiv s_{33}-s_{13} .
\end{aligned}
$$


The resulting fractional displacements due to aligned defects satisfy

$$
\left(x_{\|}-x_{\perp}\right)=\frac{\rho}{2} \sum_{i}\left\{2 F_{z i} R_{z i} \gamma_{\|}-\left(F_{x i} R_{x i}+F_{y i} R_{y i}\right) \gamma_{\perp}\right\}
$$

3.3.1. Simple defects in the hexagonal lattice. For force dipoles parallel to the hexagonal axis, we have forces $\pm(0,0, F)$ at positions $\pm(0,0, d / 2)$. This gives

$$
\left(x_{\|}-x_{\perp}\right)=\rho \gamma_{\|} F d .
$$

Similarly, for force dipoles within the basal plane, we have forces $\pm(0, F, 0)$ at positions $\pm(0, d / 2,0)$, for example. Hence

$$
\left(x_{\mid !}-x_{\perp}\right)=-\frac{\rho \gamma_{\perp}}{2} F d .
$$

If there are densities $\rho_{\| !}$along and $\rho_{\perp}$ normal to the hexagonal axis, then the overall fractional displacements are:

$$
\left(x_{\| !}-x_{\perp}\right)=\frac{F d}{2}\left(2 \rho_{\| !} \gamma_{\|}-\rho_{\perp} \gamma_{\perp}\right)
$$

There is no change in shape $\left(x_{\|}=x_{\perp}\right)$ when $\rho_{\| !}$and $\rho_{\perp}$ are related by:

$$
2 \rho_{1 \mid}\left(c_{11}+c_{12}+c_{13}\right)=\rho_{\perp}\left(c_{33}+2 c_{13}\right) \text {. }
$$

More general defects are readily treated. The simplest generalization is to represent a defect by three orthogonal dipoles described by $F_{\| !} d_{\|}, F_{\perp 1} d_{\perp 1}$ and $F_{\perp 2} d_{\perp 2}$. The result

$$
\left(x_{\| !}-x_{\perp}\right)=\left(\rho F_{\|} d_{\| !}\right) \gamma_{\| !}-\left(\frac{\rho}{2} F_{\perp 1} d_{\perp 1}+F_{\perp 2} d_{\perp 2}\right) \gamma_{\perp}
$$

has the same form as the equations of Henson and Reynolds (1965) and Reynolds (1966) used in analysing radiation damage in graphite. Their results can be expressed in the form:

$$
\left(x_{\|}-x_{\perp}\right)=P_{c} \gamma_{\|}-P_{a} \gamma_{\perp}
$$

Comparison with (10) shows that in general:

$$
\begin{aligned}
& P_{c} \equiv \rho \sum_{i} F_{z i} R_{z i} \\
& P_{a} \equiv \frac{1}{2} \rho \sum_{i}\left[F_{x i} R_{x i}+F_{y i} R_{y i}\right] .
\end{aligned}
$$

There is no need to suppose that all defects can be simply described by force dipoles. The force arrays may lack appropriate centres of inversion. The vacancy in graphite is an example. If there is no asymmetric Jahn-Teller distortion, the defect can be represented approximately by three radial forces $F$ on the nearest neighbour sites $(a, 0,0)$ and $(-a / 2, \pm \sqrt{3} a / 2,0)$. The fractional changes in dimension satisfy:

$$
\left(x_{\| !}-x_{\perp}\right)=-\frac{3}{2} \rho \gamma_{\perp} F a
$$




\section{Conclusion}

We have shown that the anisotropic changes in external dimensions of a solid due to the presence of aligned anisotropic defects can be written as simple functions of the microscopic forces due to the individual defects. Explicit formulae have been given for simpler cases involving isotropic, cubic and hexagonal lattices. It has also been possible to see how these changes vary with the position of the defect within the crystal. It can be seen directly from (6) that the only effects arise from modifications of the $\boldsymbol{R}_{i}$ at which the forces should be calculated, or in situations where the range of the forces $F_{i}$ goes beyond the crystal boundary. Neither situation is likely to be very important in practice.

\section{Acknowledgments}

I am grateful to Dr D J Bacon and Dr R Bullough for useful conversations.

\section{References}

Betti E 1872 Nuovo Cim. Ser. 2 tt 7 and 8

Henson R W and Reynolds W N 1965 Carbon 3 277-87

Love A E H 1944 A Treatise on the Mathematical Theory of Elasticity (New York: Dover)

Reynolds W N 1966 Chem. Phys. Carbon 2121

Simmons R O and Balluffi R W 1960 Phys. Rev. 11752

Temkin D E 1970 Sov. Phys.-Solid St. 111614 